FACTA UNIVERSITATIS (NIŠ)

Ser. Math. Inform. Vol. 35, No 5 (2020), 1475-1488

https://doi.org/10.22190/FUMI2005475M

\title{
NONLINEAR NEUTRAL CAPUTO-FRACTIONAL DIFFERENCE EQUATIONS WITH APPLICATIONS TO LOTKA-VOLTERRA NEUTRAL MODEL
}

\author{
Mouataz Billah Mesmouli, Abdelouaheb Ardjouni and Ahcene Djoudi
}

(C) 2020 by University of Niš, Serbia / Creative Commons Licence: CC BY-NC-ND

Abstract. In this paper, we consider a nonlinear neutral fractional difference equations. By applying Krasnoselskii's fixed point theorem, sufficient conditions for the existence of solutions are established. Also, the uniqueness of a solution is given. As an application of the main theorems, we provide the existence and uniqueness of the discrete fractional Lotka-Volterra model of neutral type. Our main results extend and generalize the results that are obtained in [6].

Key words: Existence and uniqueness; fractional difference equations; Krasnoselskii fixed point theorem; contraction operator; Arzela-Ascoli's theorem; neutral discrete fractional Lotka-Volterra model.

\section{Introduction and preliminaries}

Fractional difference equations have received a special attention during the last years. Indeed, some mathematicians have recently taken the lead to develop the qualitative properties of fractional difference equations. We recall, for instance, the study made by Atici et. al. [7], [8], [9] and Abdeljawad et. al. [1], [2] (see also [4], [12], [15], [19]-[23], [25] and reference therein) who developed the transform methods, properties of initial value problems and studied applications of these equations.

Let $\mathbb{N}_{0}=\left[0, T_{1}\right] \cap \mathbb{Z}$ where $T_{1} \in[2,+\infty) \cap \mathbb{Z}$. Alzabut, Abdeljawad and Baleanu [6] discussed the existence of solutions for the difference equation

$$
\left\{\begin{array}{l}
{ }^{c} \nabla_{0}^{\alpha} x(t)=f\left(t, x(t), x\left(t-\tau_{1}\right)\right), t \in \mathbb{N}_{0}, \\
x(t)=\psi(t), t \in\left[-\tau_{1}, 0\right] \cap \mathbb{Z},
\end{array}\right.
$$

where $\tau_{1} \in \mathbb{N}, \psi:\left[-\tau_{1}, 0\right] \cap \mathbb{Z} \rightarrow \mathbb{R}, f: \mathbb{N}_{0} \times \mathbb{R} \times \mathbb{R} \rightarrow \mathbb{R}$ and ${ }^{c} \nabla_{0}^{\alpha}$ denotes the Caputo's fractional difference of order $\alpha \in(0,1)$. By employing the Krasnoselskii fixed point theorem, the authors obtained existence results.

Received May 20, 2020; accepted November 25, 2020

2020 Mathematics Subject Classification. Primary 34A08; Secondary 34A12, 39A12. 
In this paper, we are interested in the analysis of qualitative theory of the problems of the existence and uniqueness of solutions to nonlinear neutral fractional difference equations

$$
\left\{\begin{array}{l}
{ }^{c} \nabla_{0}^{\alpha} x(t)=f\left(t, x(t), x\left(t-\tau_{1}\right),{ }^{c} \nabla_{0}^{\alpha} x\left(t-\tau_{2}\right)\right), t \in \mathbb{N}_{0}, \\
x(t)=\psi(t), t \in[-\tau, 0] \cap \mathbb{Z}
\end{array}\right.
$$

where $\tau_{1}, \tau_{2} \in \mathbb{N}, \tau=\max \left(\tau_{1}, \tau_{2}\right), \psi:[-\tau, 0] \cap \mathbb{Z} \rightarrow \mathbb{R}, f: \mathbb{N}_{0} \times \mathbb{R} \times \mathbb{R} \times \mathbb{R} \rightarrow \mathbb{R}$ and ${ }^{c} \nabla_{0}^{\alpha}$ denotes the Caputo's fractional difference of order $\alpha \in(0,1)$. To prove our main results, we employ the Krasnoselskii and Banach fixed point theorems and the Arzelá-Ascoli's theorem. Moreover, we apply the main theorems to the discrete fractional Lotka-Volterra of neutral type

$$
\left\{\begin{array}{l}
{ }^{c} \nabla_{0}^{\alpha} x(t)=x(t)\left[a(t)-b(t) x\left(t-\tau_{1}\right)-c(t)^{c} \nabla_{0}^{\alpha} x\left(t-\tau_{2}\right)\right], t \in \mathbb{N}_{0}, \\
x(t)=\psi(t), t \in[-\tau, 0] \cap \mathbb{Z},
\end{array}\right.
$$

where $a, b$ and $c$ are sequences fulfill some of the conditions described below, which are medically and biologically feasible.

Now, we present some basic definitions, notations and results of fractional difference calculus [16], [17] which are used throughout this paper. For any $\alpha, t \in \mathbb{R}$, the $\alpha$ rising function is defined by

$$
t^{\bar{\alpha}}=\frac{\Gamma(t+1)}{\Gamma(t)}, t \in \mathbb{R}-\{\ldots,-2,-1,0\}, 0^{\bar{\alpha}}=0,
$$

where $\Gamma$ is the well known Gamma function satisfying $\Gamma(\alpha+1)=\alpha \Gamma(\alpha)$.

Definition 1.1. Let $x: \mathbb{N}_{0} \rightarrow \mathbb{R}, \rho(s)=s-1, \alpha \in \mathbb{R}^{+}$and $\mu>-1$. Then

1) The nabla difference of $x$ is defined by

$$
\nabla x(t)=x(t)-x(t-1), t \in \mathbb{N}_{1}=\left[1, T_{1}\right] \cap \mathbb{Z} .
$$

2) The Riemann-Liouville's sum operator of $x$ of order $\alpha>0$ is defined by

$$
\nabla_{0}^{-\alpha} x(t)=\frac{1}{\Gamma(\alpha)} \sum_{s=1}^{t}(t-\rho(s))^{\overline{\alpha-1}}, t \in \mathbb{N}_{1} .
$$

3) The Riemann-Liouville's difference operator of $x$ of order $0<\alpha<1$ is defined by

$$
{ }^{c} \nabla_{0}^{\alpha} x(t)=\nabla_{0}^{-(1-\alpha)} \nabla x(t)=\frac{1}{\Gamma(1-\alpha)} \sum_{s=1}^{t}(t-\rho(s))^{\overline{-\alpha}} \nabla x(s), t \in \mathbb{N}_{1} .
$$

4) The power rule is defined by

$$
\nabla_{0}^{-\alpha} t^{\bar{\mu}}=\frac{\Gamma(\mu+1-\alpha)}{\Gamma(\mu+\alpha+1)} t^{\overline{\mu+\alpha}}, t \in \mathbb{N}_{1} .
$$


Let $\mathbb{N}_{-\tau}=\left[-\tau, T_{1}\right] \cap \mathbb{Z}$ where $T_{1} \in[3,+\infty) \cap \mathbb{Z}$, and $B\left(\mathbb{N}_{-\tau}, \mathbb{R}\right)$ be the Banach space of all bounded sequences with respect to the maximum norm.

Definition 1.2. A set $D$ of sequences in $B\left(\mathbb{N}_{-\tau}, \mathbb{R}\right)$ is uniformly Cauchy if for every $\epsilon>0$, there exists an integer $N^{*}$ such that $|x(t)-x(s)|<\epsilon$ whenever $t, s>N^{*}$ for any $x=\{x(n)\}$ in $D$.

The following discrete version of Arzelá-Ascoli's theorem has a crucial role in the proof of our main theorems.

Theorem 1.1. Arzelá-Ascoli's theorem A bounded, uniformly Cauchy subset $D$ of $B\left(\mathbb{N}_{-\tau}, \mathbb{R}\right)$ is relatively compact.

The proof of the main theorem is achieved by employing the following fixed point theorem.

Theorem 1.2. Krasnoselskii's fixed point theorem [10] Let $D$ be a nonempty, closed, convex and bounded subset of a Banach space $(X,\|\cdot\|)$. Suppose that $A_{1}: D \rightarrow X$ and $A_{2}: D \rightarrow X$ are two operators such that

(i) $A_{1}$ is a contraction,

(ii) $A_{2}$ is continuous and $A_{2}(D)$ resides in a compact subset of $X$,

(iii) for any $x, y \in D, A_{1} x+A_{2} y \in D$.

Then the operator $A_{1}+A_{2}$ has a fixed point $x \in D$.

\section{Existence and uniqueness of solutions}

In this section, we give the equivalence of the problem (1.2). So, by an alternative way used in [3], [5] and [14], we turn the problem (1.2) into an equivalent equation, then, the solvability of this equivalent equation implies the existence and uniqueness of solution to the problem (1.2).

Lemma 2.1. $x$ denotes a solution of the equation (1.2) if and only if it admits the following representation

$$
x(t)=\psi(0)+\frac{1}{\Gamma(\alpha)} \sum_{s=1}^{t}(t-\rho(s))^{\overline{\alpha-1}} z_{x}(s),
$$

where $z_{x}(t)={ }^{c} \nabla_{0}^{\alpha} x(t)$ and $x(t)=\psi(t), t \in[-\tau, 0] \cap \mathbb{Z}$.

Proof. By the same way used in [3], we get for $t \in \mathbb{N}_{-\tau}$, the initial value problem (1.2) is equivalent to the following equation

$(2.2) x(t)=\psi(0)+\frac{1}{\Gamma(\alpha)} \sum_{s=1}^{t}(t-\rho(s))^{\overline{\alpha-1}} f\left(t, x(s), x\left(s-\tau_{1}\right),{ }^{c} \nabla_{0}^{\alpha} x\left(s-\tau_{2}\right)\right)$. 
By the techniques used in [5] and [14], let

$$
z_{x}(t)={ }^{c} \nabla_{0}^{\alpha} x(t) \text { and } x(t)=\psi(t) \text { for } t \in[-\tau, 0] \cap \mathbb{Z} .
$$

Then, the equation (2.2) is equivalent to the equation (2.1), with

$$
z_{x}(t)=f\left(t, x(t), x\left(t-\tau_{1}\right), z_{x}\left(t-\tau_{2}\right)\right) .
$$

We prove our main results under the following assumptions

(A1) For $t \in \mathbb{N}_{-\tau}$,

$$
\begin{aligned}
z_{x}(t) & =f\left(t, x(t), x\left(t-\tau_{1}\right), z_{x}\left(t-\tau_{2}\right)\right) \\
& =f_{1}(t, x(t))+f_{2}\left(t, x(t), x\left(t-\tau_{1}\right)\right)+f_{3}\left(t, x, z_{x}\left(t-\tau_{2}\right)\right),
\end{aligned}
$$

where $f_{1}, f_{2}$ and $f_{3}$ are Lipschitz functions with Lipschitz constants $L_{f_{i}}$, $i=1,2,3$, with $L_{f_{3}}<1$.

(A2) For $t \in \mathbb{N}_{-\tau}$,

$$
\begin{aligned}
\left|f_{1}(t, u(t))\right| & \leq M_{1}|u(t)| \\
\left|f_{2}(t, u(t), v(t))\right| & \leq M_{2}|u(t)||v(t)|, \\
\left|f_{3}(t, u(t), v(t))\right| & \leq M_{3}|u(t)||v(t)|,
\end{aligned}
$$

for any positive numbers $M_{i}, i=1,2,3$.

Define the set

$$
D=\left\{u \in B\left(\mathbb{N}_{-\tau}, \mathbb{R}\right),\|u\| \leq r, u(t)=\psi(t) \text { for } t \in[-\tau, 0] \cap \mathbb{Z}\right\},
$$

where $r$ satisfies

$$
|\psi(0)|+\frac{M_{1} r+M_{2} r^{2}+M_{3} L r^{2}}{\Gamma(\alpha)} C(\alpha) \leq r,
$$

and $C(\alpha)=\frac{\Gamma\left(T_{1}+\alpha\right)}{\alpha \Gamma\left(T_{1}\right)}$ is a positive constant depending on the order $\alpha$ and satisfies the inequality

$$
L_{f_{1}} C(\alpha)<\Gamma(\alpha) .
$$

Lemma 2.2. Suppose that the assumption (A1) holds. Then, for $t \in \mathbb{N}_{-\tau}, z_{x}$ satisfies the following inequality

$$
\left|z_{x}(t)-z_{y}(t)\right| \leq L\|x-y\| \text { for all } x, y \in B\left(\mathbb{N}_{-\tau}, \mathbb{R}\right),
$$

where

$$
L=\frac{L_{f_{1}}+2 L_{f_{2}}+L_{f_{3}}}{1-L_{f_{3}}} .
$$


Proof. For all $x, y \in B\left(\mathbb{N}_{-\tau}, \mathbb{R}\right)$, since (A1) holds, then

$$
\begin{aligned}
& \left|z_{x}(t)-z_{y}(t)\right| \\
\leq & L_{f_{1}}|x(t)-y(t)|+L_{f_{2}}|x(t)-y(t)|+L_{f_{2}}\left|x\left(t-\tau_{1}\right)-y\left(t-\tau_{1}\right)\right| \\
& +L_{f_{3}}|x(t)-y(t)|+L_{f_{3}}\left|z_{x}\left(t-\tau_{2}\right)-z_{y}\left(t-\tau_{2}\right)\right| \\
\leq & \left(L_{f_{1}}+2 L_{f_{2}}+L_{f_{3}}\right)\|x-y\|+L_{f_{3}}\left\|z_{x}-z_{y}\right\| .
\end{aligned}
$$

Thus,

$$
\left|z_{x}(t)-z_{y}(t)\right| \leq \frac{L_{f_{1}}+2 L_{f_{2}}+L_{f_{3}}}{1-L_{f_{3}}}\|x-y\| .
$$

Now, to apply Krasnoselskii's fixed point 1.2 , by Lemma 2.1 can define the operators $A_{1}$ and $A_{2}$ on $D$ by

$$
\left(A_{1} x\right)(t)=\psi(0)+\frac{1}{\Gamma(\alpha)} \sum_{s=1}^{t}(t-\rho(s))^{\overline{\alpha-1}} f_{1}(s, x(s)),
$$

and

$$
\begin{aligned}
\left(A_{2} x\right)(t)= & \frac{1}{\Gamma(\alpha)} \sum_{s=1}^{t}(t-\rho(s))^{\overline{\alpha-1}} f_{2}\left(s, x(s), x\left(s-\tau_{1}\right)\right) \\
& +\frac{1}{\Gamma(\alpha)} \sum_{s=1}^{t}(t-\rho(s))^{\overline{\alpha-1}} f_{3}\left(s, x(s), z_{x}\left(s-\tau_{2}\right)\right) .
\end{aligned}
$$

It is clear that $x$ is a solution of (1.2) if it is a fixed point of the operator $A=A_{1}+A_{2}$.

Theorem 2.1. Let conditions (A1), (A2), (2.5) and (2.6) hold. Then, the equation (1.2) has a solution in the set D.

Proof. From the assumptions on the set $D$, one can easily see that $D$ is a nonempty, closed, convex and bounded set.

Step 1. We prove that the $A_{1}$ defined by (2.7) is contraction. We can easily see that for $x, y \in D$

$$
\begin{aligned}
& \left|\left(A_{1} x\right)(t)-\left(A_{1} y\right)(t)\right| \\
\leq & \frac{1}{\Gamma(\alpha)} \sum_{s=1}^{t}(t-\rho(s))^{\overline{\alpha-1}}\left|f_{1}(s, x(s))-f_{1}(s, y(s))\right| \\
\leq & L_{f_{1}} \frac{1}{\Gamma(\alpha)} \sum_{s=1}^{t}(t-\rho(s))^{\overline{\alpha-1}}|x(s)-y(s)| \\
\leq & \frac{L_{f_{1}}}{\Gamma(\alpha)}\|x-y\| \sum_{s=1}^{t}(t-\rho(s))^{\overline{\alpha-1}} .
\end{aligned}
$$


By virtue of $(1.4),(1.5),(1.7)$ and since $(t-0)^{\overline{0}}=1$, one can see that

$$
\sum_{s=1}^{t}(t-\rho(s))^{\overline{\alpha-1}}(t-0)^{\overline{0}}=\Gamma(\alpha) \nabla_{0}^{-\alpha}(t-0)^{\overline{0}}=\frac{\Gamma(t+\alpha)}{\alpha \Gamma(t)} .
$$

Therefore,

$$
\left|\left(A_{1} x\right)(t)-\left(A_{1} y\right)(t)\right| \leq \frac{C(\alpha)}{\Gamma(\alpha)} L_{f_{1}}\|x-y\|, t \leq T_{1} .
$$

By the assumption (2.6), we conclude that $A_{1}$ is contraction mapping on $D$.

Furthermore, we obtain for $x \in D$

$$
\begin{aligned}
& \left|\left(A_{1} x\right)(t)+\left(A_{2} x\right)(t)\right| \\
\leq & \left|\psi(0)+\frac{1}{\Gamma(\alpha)} \sum_{s=1}^{t}(t-\rho(s))^{\overline{\alpha-1}} f_{1}(s, x(s))\right| \\
& +\left|\frac{1}{\Gamma(\alpha)} \sum_{s=1}^{t}(t-\rho(s))^{\overline{\alpha-1}} f_{2}\left(s, x(s), x\left(s-\tau_{1}\right)\right)\right| \\
& +\left|\frac{1}{\Gamma(\alpha)} \sum_{s=1}^{t}(t-\rho(s))^{\overline{\alpha-1}} f_{3}\left(s, x(s), z_{x}\left(s-\tau_{2}\right)\right)\right| \\
\leq & |\psi(0)|+\frac{M_{1}\|x\|+M_{2}\|x\|^{2}+M_{3} L\|x\|^{2}}{\Gamma(\alpha)} \sum_{s=1}^{t}(t-\rho(s))^{\overline{\alpha-1}} \\
\leq & |\psi(0)|+\frac{M_{1} r+M_{2} r^{2}+M_{3} L r^{2}}{\Gamma(\alpha)} C(\alpha) \\
\leq & r,
\end{aligned}
$$

which implies that $A_{1} x+A_{2} x \in D$. For $x \in D$, we also get

$$
\begin{aligned}
\left|\left(A_{2} x\right)(t)\right| \leq & \left|\frac{1}{\Gamma(\alpha)} \sum_{s=1}^{t}(t-\rho(s))^{\overline{\alpha-1}} f_{2}\left(s, x(s), x\left(s-\tau_{1}\right)\right)\right| \\
& +\left|\frac{1}{\Gamma(\alpha)} \sum_{s=1}^{t}(t-\rho(s))^{\overline{\alpha-1}} f_{3}\left(s, x(s), z_{x}\left(s-\tau_{2}\right)\right)\right| \\
\leq & \frac{M_{2}\|x\|^{2}+M_{3} L\|x\|^{2}}{\Gamma(\alpha)} \sum_{s=1}^{t}(t-\rho(s))^{\overline{\alpha-1}} \\
\leq & \frac{M_{2} r^{2}+M_{3} L r^{2}}{\Gamma(\alpha)} C(\alpha) \\
\leq & r
\end{aligned}
$$

which implies that $A_{2}(D) \subset D$. 
Step 2. We prove that $A_{2}$ is continuous. Let a sequence $x_{n}$ converge to $x$. Taking the norm of $A_{2} x_{n}-A_{2} x$, we have

$$
\begin{aligned}
& \left|\left(A_{2} x_{n}\right)(t)-\left(A_{2} x\right)(t)\right| \\
\leq & \frac{1}{\Gamma(\alpha)} \sum_{s=1}^{t}(t-\rho(s))^{\overline{\alpha-1}}\left|f_{2}\left(s, x_{n}(s), x_{n}\left(s-\tau_{1}\right)\right)-f_{2}\left(s, x(s), x\left(s-\tau_{1}\right)\right)\right| \\
& +\frac{1}{\Gamma(\alpha)} \sum_{s=1}^{t}(t-\rho(s))^{\overline{\alpha-1}}\left|f_{3}\left(s, x_{n}(s), z_{x_{n}}\left(s-\tau_{2}\right)\right)-f_{3}\left(s, x(s), z_{x}\left(s-\tau_{2}\right)\right)\right| \\
= & \frac{C(\alpha)}{\Gamma(\alpha)}\left(2 L_{f_{2}}+L_{f_{3}}+L_{f_{3}} L\right)\left\|x_{n}-x\right\| .
\end{aligned}
$$

Then, we conclude that whenever $x_{n} \rightarrow x, A_{2} x_{n} \rightarrow A_{2} x$. This proves the continuity of $A_{2}$. To prove that $A_{2}(D)$ resides in a compact subset of $B\left(\mathbb{N}_{-\tau}, \mathbb{R}\right)$, i.e., $A_{2}(D)$ is a relatively compact subset. We let $t_{1} \leq t_{2} \leq T_{1}$ to get

$$
\begin{aligned}
& \left|\left(A_{2} x\right)\left(t_{2}\right)-\left(A_{2} x\right)\left(t_{1}\right)\right| \\
\leq & \frac{1}{\Gamma(\alpha)} \mid \sum_{s=1}^{t_{2}}\left(t_{2}-\rho(s)\right)^{\overline{\alpha-1}} f_{2}\left(s, x(s), x\left(s-\tau_{1}\right)\right) \\
& -\sum_{s=1}^{t_{1}}\left(t_{1}-\rho(s)\right)^{\overline{\alpha-1}} f_{2}\left(s, x(s), x\left(s-\tau_{1}\right)\right) \mid \\
& +\frac{1}{\Gamma(\alpha)} \mid \sum_{s=1}^{t_{2}}\left(t_{2}-\rho(s)\right)^{\overline{\alpha-1}} f_{3}\left(s, x(s), z_{x}\left(s-\tau_{2}\right)\right) \\
& -\sum_{s=1}^{t_{1}}\left(t_{1}-\rho(s)\right)^{\overline{\alpha-1}} f_{3}\left(s, x(s), z_{x}\left(s-\tau_{2}\right)\right) \mid \\
\leq & \frac{1}{\Gamma(\alpha)} \sum_{s=1}^{t_{1}}\left|\left(t_{2}-\rho(s)\right)^{\overline{\alpha-1}}-\left(t_{1}-\rho(s)\right)^{\overline{\alpha-1}}\right|\left|f_{2}\left(s, x(s), x\left(s-\tau_{1}\right)\right)\right| \\
& +\frac{1}{\Gamma(\alpha)} \sum_{s=t_{1}+1}^{t_{2}}\left|\left(t_{2}-\rho(s)\right)^{\overline{\alpha-1}}\right|\left|f_{2}\left(s, x(s), x\left(s-\tau_{1}\right)\right)\right| \\
& +\frac{1}{\Gamma(\alpha)} \sum_{s=1}^{t_{1}}\left|\left(t_{2}-\rho(s)\right)^{\overline{\alpha-1}}-\left(t_{1}-\rho(s)\right)^{\overline{\alpha-1}}\right|\left|f_{3}\left(s, x(s), z_{x}\left(s-\tau_{2}\right)\right)\right| \\
& +\frac{1}{\Gamma(\alpha)} \sum_{s=t_{1}+1}^{t_{2}}\left|\left(t_{2}-\rho(s)\right)^{\overline{\alpha-1}}\right|\left|f_{3}\left(s, x(s), z_{x}\left(s-\tau_{2}\right)\right)\right| .
\end{aligned}
$$


By the assumption (A2) and Lemma 2.2, we obtain

$$
\begin{aligned}
& \left|\left(A_{2} x\right)\left(t_{2}\right)-\left(A_{2} x\right)\left(t_{1}\right)\right| \\
\leq & \left(M_{2} r^{2}+M_{3} L r^{2}\right)\left[\frac{1}{\Gamma(\alpha)} \sum_{s=1}^{t_{1}}\left|\left(t_{2}-\rho(s)\right)^{\overline{\alpha-1}}-\left(t_{1}-\rho(s)\right)^{\overline{\alpha-1}}\right|\right. \\
& \left.+\frac{1}{\Gamma(\alpha)} \sum_{s=t_{1}+1}^{t_{2}}\left|\left(t_{2}-\rho(s)\right)^{\overline{\alpha-1}}\right|\right] .
\end{aligned}
$$

By using (1.5), we get

$$
\begin{aligned}
& \left|\left(A_{2} x\right)\left(t_{2}\right)-\left(A_{2} x\right)\left(t_{1}\right)\right| \\
\leq & \left(M_{2} r^{2}+M_{3} L r^{2}\right)\left(\left(t_{2}\right)^{\overline{0}}-\left(t_{1}\right)^{\overline{0}}+\left(t_{2}-t_{1}\right)^{\overline{0}}\right) .
\end{aligned}
$$

From (1.7), it follows that

$$
\begin{aligned}
& \left|\left(A_{2} x\right)\left(t_{2}\right)-\left(A_{2} x\right)\left(t_{1}\right)\right| \\
\leq & \frac{\left(M_{2} r^{2}+M_{3} L r^{2}\right)}{\Gamma(\alpha+1)}\left(\nabla_{0}^{-\alpha}\left(t_{2}-0\right)^{\overline{0}}-\nabla_{0}^{-\alpha}\left(t_{1}-0\right)^{\overline{0}}+\nabla_{t_{1}}^{-\alpha}\left(t_{2}-t_{1}\right)^{\overline{0}}\right) .
\end{aligned}
$$

This implies that $A_{2}(D)$ is uniformly bounded subset of $B\left(\mathbb{N}_{-\tau}, \mathbb{R}\right)$. Thus, by virtue of the discrete Arzelá-Ascoli's theorem 1.1, we conclude that $A_{2}$ is compact.

Step 3. It remains to show that for any $x, y \in D$, we have $A_{1} x+A_{2} y \in D$. If $x, y \in D$, then we have

$$
\begin{aligned}
& \left|\left(A_{1} x\right)(t)+\left(A_{2} y\right)(t)\right| \\
\leq & \left|\psi(0)+\frac{1}{\Gamma(\alpha)} \sum_{s=1}^{t}(t-\rho(s))^{\overline{\alpha-1}} f_{1}(s, x(s))\right| \\
& +\left|\frac{1}{\Gamma(\alpha)} \sum_{s=1}^{t}(t-\rho(s))^{\overline{\alpha-1}} f_{2}\left(s, y(s), y\left(s-\tau_{1}\right)\right)\right| \\
& +\left|\frac{1}{\Gamma(\alpha)} \sum_{s=1}^{t}(t-\rho(s))^{\overline{\alpha-1}} f_{3}\left(s, y(s), z_{y}\left(s-\tau_{2}\right)\right)\right| \\
\leq & |\psi(0)|+\frac{M_{1}\|x\|+M_{2}\|y\|^{2}+M_{3} L\|y\|^{2}}{\Gamma(\alpha)} \sum_{s=1}^{t}(t-\rho(s))^{\overline{\alpha-1}} \\
\leq & |\psi(0)|+\frac{M_{1} r+M_{2} r^{2}+M_{3} L r^{2}}{\Gamma(\alpha)} C(\alpha) \\
\leq & r,
\end{aligned}
$$

which implies that $A_{1} x+A_{2} y \in D$.

By employing the Krasnoselskii fixed point theorem, we conclude that there exists $x \in D$ such that $x=A x=A_{1} x+A_{2} x$ which is a fixed point of $A$. Hence, the equation (1.2) has at least one solution in $D$. 
Remark 2.1. Note that, when $f_{3} \equiv 0$ Theorem 2.1 becomes the same Theorem 3 in [6], and this confirms the generality of the results.

It is worth noting that, the authors in [6] stated that they studied the uniqueness of solutions for the equation (1.2), but in reality they did not, because Krasnoselskii's theorem only gives us the existence of solutions, it may be only a written error. So, in this paper, we will study the uniqueness of solutions as well.

Theorem 2.2. Let conditions (A1), (A2), (2.5) and

$$
\frac{C(\alpha)}{\Gamma(\alpha)}\left(L_{f_{1}}+2 L_{f_{2}}+L_{f_{3}}+L_{f_{3}} L\right)<1,
$$

hold. Then, the equation (1.2) has a unique solution in D.

Proof. Since the equation (1.2) is equivalent to (2.1), for $x \in D$ define

$$
A x=A_{1} x+A_{2} x .
$$

Step 1. We must prove that $A$ maps $D$ into itself, then by the condition (2.5) and the same way in $(2.9)$

$$
\begin{aligned}
|(A x)(t)| & =\left|\left(A_{1} x\right)(t)+\left(A_{2} x\right)(t)\right| \\
& \leq|\psi(0)|+\frac{M_{1} r+M_{2} r^{2}+M_{3} L r^{2}}{\Gamma(\alpha)} C(\alpha) \\
& \leq r,
\end{aligned}
$$

which implies that $A x \in D$.

Step 2. We prove that $A$ is contraction. We can see that for $x, y \in D$

$$
\begin{aligned}
& |(A x)(t)-(A y)(t)| \\
\leq & \frac{L_{f_{1}}}{\Gamma(\alpha)}\|x-y\| \sum_{s=1}^{t}(t-\rho(s))^{\overline{\alpha-1}}+\frac{2 L_{f_{2}}}{\Gamma(\alpha)}\|x-y\| \sum_{s=1}^{t}(t-\rho(s))^{\overline{\alpha-1}} \\
& +\frac{\left(L_{f_{3}}+L_{f_{3}} L\right)}{\Gamma(\alpha)}\|x-y\| \sum_{s=1}^{t}(t-\rho(s))^{\overline{\alpha-1}} .
\end{aligned}
$$

Therefore,

$$
|(A x)(t)-(A y)(t)| \leq \frac{C(\alpha)}{\Gamma(\alpha)}\left(L_{f_{1}}+2 L_{f_{2}}+L_{f_{3}}+L_{f_{3}} L\right)\|x-y\|, t \leq T_{1} .
$$

By the assumption (2.10), we conclude that $A$ is contraction mapping on $D$.

By employing the Banach fixed point theorem, we conclude that there exists a unique $x \in D$ such that $x=A x$ which is a unique fixed point of $A$. Hence, the equation (1.2) has a unique solution in $D$. 
Remark 2.2. Note that, when $f_{3} \equiv 0$ Theorem 2.2 gives the uniqueness of the solution of the equation (1.1).

Now, we can replace the assumptions (A2) and (2.5) by the following, which provide us the existence and uniqueness too.

$(\overline{\mathbf{A 2}})$ For $t \in \mathbb{N}_{-\tau}$, we assume that $f_{1}(t, 0)=f_{2}(t, 0,0)=f_{3}(t, 0,0) \equiv 0$ and

$$
|\psi(0)|+\frac{\left(L_{f_{1}}+2 L_{f_{2}}+L_{f_{3}}(1+L)\right) r}{\Gamma(\alpha)} C(\alpha) \leq r .
$$

Then we get the following theorems.

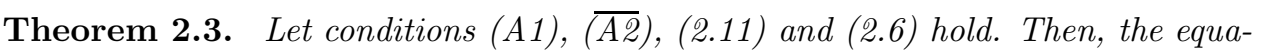
tion (1.2) has a solution in the set $D$.

Proof. The proof is based on the following estimation, since $f_{1}, f_{2}$ and $f_{3}$ satisfy the assumptions (A1) and $(\overline{\mathrm{A} 2})$, then

$$
\begin{aligned}
\left|f_{1}(t, x(t))\right| & =\left|f_{1}(t, x(t))-f_{1}(t, 0)\right| \\
& \leq L_{f_{1}}\|x\| \\
\left|f_{2}\left(t, x(t), x\left(t-\tau_{1}\right)\right)\right| & =\left|f_{2}\left(t, x(t), x\left(t-\tau_{1}\right)\right)-f_{2}(t, 0,0)\right| \\
& \leq 2 L_{f_{2}}\|x\|,
\end{aligned}
$$

and

$$
\begin{aligned}
\left|f_{3}\left(t, x(t), z_{x}\left(t-\tau_{2}\right)\right)\right| & =\left|f_{3}\left(t, x(t), z_{x}\left(t-\tau_{2}\right)\right)-f_{3}(t, 0,0)\right| \\
& \leq L_{f_{3}}\left(\|x\|+\left\|z_{x}\right\|\right) \\
& \leq L_{f_{3}}(\|x\|+L\|x\|) \\
& =L_{f_{3}}(1+L)\|x\| .
\end{aligned}
$$

The remaining steps of the proof are the same as in Theorem 2.1.

Theorem 2.4. Let conditions (A1), (T2), (2.11) and (2.10) hold. Then, the equation (1.2) has a unique solution in $D$.

Proof. The steps of the proof is given by the same way in Theorem 2.2.

Remark 2.3. The results of this paper can be carried out for the equation

$$
\left\{\begin{array}{l}
\nabla_{0}^{\alpha} x(t)=f\left(t, x(t), x\left(t-\tau_{1}\right),{ }^{c} \nabla_{0}^{\alpha} x\left(t-\tau_{2}\right)\right), t \in \mathbb{N}_{2}=\left[2, T_{1}\right] \cap \mathbb{Z}, \\
x(t)=\psi(t), t \in[-\tau, 1] \cap \mathbb{Z},
\end{array}\right.
$$

where $\tau_{1}, \tau_{2} \in \mathbb{N}, \tau=\max \left(\tau_{1}, \tau_{2}\right), T_{1} \in[4,+\infty) \cap \mathbb{Z}, \psi:[-\tau, 1] \cap \mathbb{Z} \rightarrow \mathbb{R}, f: \mathbb{N}_{0} \times \mathbb{R} \times \mathbb{R} \rightarrow \mathbb{R}$ and $\nabla_{0}^{\alpha}$ denotes the Riemann-Liouville's fractional difference of order $\alpha \in(0,1)$. The solution of the equation (2.12) has the form

$$
x(t)=\frac{t^{\overline{\alpha-1}}}{\Gamma(\alpha)} \psi(1)+\frac{1}{\Gamma(\alpha)} \sum_{s=2}^{t}(t-\rho(s))^{\overline{\alpha-1}} z_{x}(s) .
$$




\section{Discrete fractional Lotka-Volterra model of neutral type}

Because it is very interesting to study the neutral delay population model. So, the Lotka-Volterra model has been extensively investigated by many authors see ([6], [9], [13], [11], [18], [24]) and others, through different approaches. But, all the above works studied the Lotka-Volterra model, or the neutral model with integer order. Then, there is no literature on the type of discrete neutral fractional LotkaVolterra model.

In this section, we employ Theorems 2.1 and 2.2 to prove the existence and uniqueness results for the solutions of (1.3), that represents an interspecific competition in single species with $\tau$ denotes the maturity time period.

For a bounded sequence $u$ on $\mathbb{N}_{0}$, we define $u^{+}$and $u^{-}$as follows

$$
u^{-}=\inf _{t \in \mathbb{N}_{0}} u(t) \text { and } u^{+}=\sup _{t \in \mathbb{N}_{0}} u(t),
$$

and denote

$$
\begin{aligned}
f_{1}(t, x(t)) & =a(t) x(t), \\
f_{2}\left(t, x(t), x\left(t-\tau_{1}\right)\right) & =-b(t) x(t) x\left(t-\tau_{1}\right), \\
f_{3}\left(t, x, z_{x}\left(t-\tau_{2}\right)\right) & =-c(t) x(t) z_{x}\left(t-\tau_{2}\right),
\end{aligned}
$$

where the coefficients $a, b$ and $c$ satisfy the boundedness relations

$$
a^{-} \leq a(t) \leq a^{+}, b^{-} \leq b(t) \leq b^{+}, c^{-} \leq c(t) \leq c^{+} .
$$

From the conditions (A1) and $(\overline{\mathrm{A} 2})$, it is easy to see that

$$
L_{f_{1}}=a^{+}, L_{f_{2}}=r b^{+}, L_{f_{3}}=r c^{+} L,
$$

and

$$
M_{1}=L_{f_{1}}, M_{2}=b^{+}, M_{3}=c^{+}
$$

Theorem 3.1. Let conditions (2.5), (2.6) and

$$
L_{f_{3}}=r c^{+} L<1
$$

hold. Then, the model (1.3) has a solution in the set D.

Theorem 3.2. Let condition (2.5), (2.10) and

$$
L_{f_{3}}=r c^{+} L<1
$$

hold. Then, the model (1.3) has a unique solution in the set D. 
Remark 3.1. The above theorems can be extended to $n$ species neutral competitive Lotka-Volterra system of the form

$$
\left\{\begin{array}{l}
{ }^{c} \nabla_{0}^{\alpha} x_{i}(t)=x_{i}(t)\left[a_{i}(t)-\sum_{j=1}^{n} b_{i j}(t) x\left(t-\tau_{i j}\right)-\sum_{j=1}^{n} c_{i j}(t)^{c} \nabla_{0}^{\alpha} x\left(t-\tau_{i j}\right)\right], t \in \mathbb{N}_{0}, \\
x_{i}(t)=\psi_{i}(t), t \in[-\tau, 0] \cap \mathbb{Z},
\end{array}\right.
$$

where $\tau_{i j} \in \mathbb{N}, \tau=\max _{1 \leq i, j \leq n} \tau_{i j}, \alpha \in(0,1), \psi_{i}:[-\tau, 0] \cap \mathbb{Z} \rightarrow \mathbb{R}, a^{-} \leq a_{i}(t) \leq a^{+}$, $b^{-} \leq b_{i j}(t) \leq b^{+}, c^{-} \leq c_{i j}(t) \leq c^{+}, i=1,2, \ldots, n$.

Acknowledgement. The authors would like to thank the anonymous referee for his/her valuable comments and good advice.

\section{REF EREN C ES}

1. T. Abdeljawad, F. M. Atici: On the definitions of nabla fractional operators. Abstr. Appl. Anal. 2012 (2012), 1-13.

2. T. AbDeljawad, D. Baleanu: Fractional differences and integration by Parts. J. Comput. Anal. Appl. 13(5) (2011), 574-582.

3. T. ABDELJAWAD: On delta and nabla Caputo fractional differences and dual identities. Discrete Dyn. Nat. Soc. 2013 (2013), 1-12.

4. B. Ahmad, D. Garout, S. K. Ntouyas, A. Alsaedi: Caputo fractional differential inclusions of arbitrary order with nonlocal integro-multipoint boundary conditions. Miskolc Mathematical Notes 20(2) (2019), 683-699.

5. A. Ardjouni, A. Djoudi: Initial-value problems for nonlinear hybrid implicit Caputo fractional differential equations. Malaya Journal of Matematik 7(2) (2019), 314-317.

6. J. Alzabut, T. Abdeljawad, D. Baleanu: Nonlinear delay fractional difference equations with applications on discrete fractional Lotka-Volterra competition model. J. Computational Analysis and Applications 25(5) (2018), 889-898.

7. F. M. Atici, P. W. Eloe: A transform method in discrete fractional calculus. Int. J. Difference Equ. 2(2) (2007), 165-176.

8. F. M. Atici, P. W. Eloe: Discrete fractional calculus with the nabla operator. Electron. J. Qual. Theory Differ. Equ. 2009(3) (2009), 1-12.

9. F. M. AtıcI, S. ŞEngüL: Modeling with factorial difference equations. J. Math. Anal. Appl. 369(1) (2010), 1-9.

10. T. A. Burton: A fixed point theorem of Krasnoselskii fixed point theorem. Appl. Math. Lett. 11 (1998), 85-88.

11. F. Capone, R. D. Luca, S. Rionero: On the stability of non-autonomous perturbed Lotka-Volterra models. Appl. Math. Comp. 219(12) (2013), 6868-6881.

12. A. Cuspilici, P. Monforte, M. A. Ragusa: Study of Saharan dust influence on PM10 measures in Sicily from 2013 to 2015. Ecological Indicators 76 (2017), 297-303.

13. F. D. Chen, X. X. CHen: The n-competing Volterra-Lotka almost periodic systems with grazing rates. J. Biomath. 18(4) (2003), 411-416. 
14. J. Dong, Y. Feng, J. JiAng: A note on implicit fractional differential equations. Mathematica Aeterna 7(3) (2017), 261-267.

15. A. Duro, V. Piccione, M. A. Ragusa, V. Veneziano: New Enviromentally Sensitive Patch Index - ESPI - for MEDALUS protocol. AIP Conference Proceedings 1637 (2014), 305-312.

16. L. Erbe, C. S. Goodrich, B. JiA, A. Peterson: Survey of the qualitative properties of fractional difference operators: monotonicity, convexity and asymptotic behavior of solutions. Adv. Differ. Equ. 2016(43) (2016), 1-31.

17. C. Goodrich, A. Peterson: Discrete Fractional Calculus. Springer 2015.

18. K. Gopalsamy, P. X. Weng: Feedback regulation of logistic growth. Int. J. Math. Sci. 16(1) (1993), 177-192.

19. E. Guariglia: Fractional Derivative of the Riemann Zeta Function. In: Fractional Dynamics, C. Cattani, H.M. Srivastava, X.-Y. Yang (Eds.), De Gruyter, 2015, pp. $357-368$.

20. E. GuARIglia: Riemann zeta fractional derivative - functional equation and link with primes. Advances in Difference Equations, 2019 (2019), 261.

21. E. Guariglia, S. Silvestrov: A functional equation for the Riemann zeta fractional derivative. AIP Conference Proceedings 1798 (2017), 020063.

22. S. Hasan, M. Al-Smadi, A. Freinet, S. Momani: Two computational approaches for solving a fractional obstacle system in Hilbert space. Adv. Differ. Equ. 2019 (2019), 55 .

23. C. LI, X. DAO, P. GuO: Fractional derivatives in complex planes. Nonlinear Analysis: Theory, Methods \& Applications, 71 (2009), 1857-1869.

24. J. X. LI, J. R. YAN: Persistence for Lotka-Volterra patch-system with time delay. Nonlinear Anal. 9(2) (2008), 490-499.

25. M. D. Ortigueira, F. Coito: From differences to derivatives. Fract. Calc. Appl. Anal. 7(4) (2004), 459-471.

Mouataz Billah Mesmouli

Faculty of Sciences, Department of Mathematics

University of Ha'il

Kingdom of Saudi Arabia

mesmoulimouataz@hotmail.com, m.mesmouli@uoh.edu.sa

Abdelouaheb Ardjouni

Faculty of Sciences and Technology

Department of Mathematics and Informatics

University of Souk-Ahras

P.O. Box 1553

Souk-Ahras, 41000, Algeria

abd_ardjouni@yahoo.fr 
Ahcene Djoudi

Faculty of Sciences, Department of Mathematics

University of Annaba

P.O. Box 12

Annaba 23000, Algeria

adjoudi@yahoo.com 